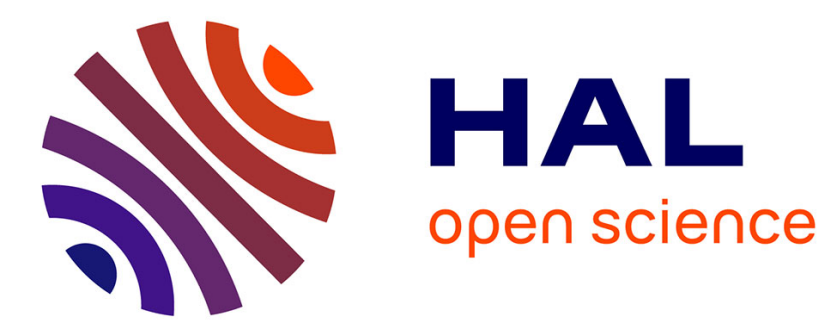

\title{
Le bas-relief de Saint-Julien-lès-Martigues (Bouches-du-Rhône)
}

\author{
Fernand Benoit
}

\section{To cite this version:}

Fernand Benoit. Le bas-relief de Saint-Julien-lès-Martigues (Bouches-du-Rhône). Gallia Fouilles et monuments archéologiques en France métropolitaine, 1948, 6 (1), pp.171-175. 10.3406/galia.1948.2069 . hal-01920867

\section{HAL Id: hal-01920867 https://hal.science/hal-01920867}

Submitted on 27 Feb 2020

HAL is a multi-disciplinary open access archive for the deposit and dissemination of scientific research documents, whether they are published or not. The documents may come from teaching and research institutions in France or abroad, or from public or private research centers.
L'archive ouverte pluridisciplinaire HAL, est destinée au dépôt et à la diffusion de documents scientifiques de niveau recherche, publiés ou non, émanant des établissements d'enseignement et de recherche français ou étrangers, des laboratoires publics ou privés.

\section{(이) $\$$}

Distributed under a Creative Commons Attribution - NonCommercial - NoDerivatives $\mid 4.0$ 


\section{NOTES ET INFORMATIONS}

\section{NOTES}

\author{
I.E: B.SS-RELILF

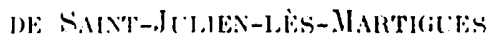 \\ (Bu(c):11:S-1)(-RHONE)
}

Ia paroi extricieme du mur du rhome de La chapelle Saint-Julien-les-Martigues est constituie, sur sa face nord, par les restes d'un imporlant monument fuméraire d'ipopue romaine, dont la partie gatuhe est masquere par to mur d'une pelite sacristie, qui vient s'appuyel sul tr relief. Le nonmment est encastré dans lo mur même de léglise: le répei a cité délouré en stivant lo profil des mules antiques (fig. 1). Il comprend six assises superposios, on grand appareil de pierre de la comronne. dont les carriores, exploitres depuis la période hellénique, ont ate utilisces dans la region marseillaise ¿ lépogue momaine: deux assises de mur servant do soubassement: relle du haut mesure $0 \mathrm{~m}$. 52 de hauteur: celle du bas, enfoncie en terre de 0 m. 15 seulement. est visible sur 0 m. 10 onviron; une assise de pierere mouluréc. haute de $0 \mathrm{~m}$. 5o aver plate-bande of large dourine en sallie de 0 m. 15) sur le relief, dont le relour a angle droit semble penétrer dans la muraille de léglise: et enfin lrois assises de grands boes sculptés, hauls de 0 m. 53 ì 0 m. 55. comportant le relief (fig. 2).

Celui-ci représente, ainsi gue lia inlerpréte .I. Ch. Picard 1 , un groupe de six persomages plus hatuls que nat-

(1) Ch. Picand, C.R.I.I., 1943, p. 439. A1tres pholos du relief dans Espéraxdice. I, 12.4: I. de Gérin-RICARD, Provincia, 1939, p. 9\%. V. la bibl. dans F.O.R., V, 153. ture 2, les deux défunts assis entourés des membres de leur famille, encadrés par les Dioscures aver leur cheval. C'est la une sculpture appartenant a une haute époque, par les détails de sa technique (qui l'apparente à l'art des stèles sculyties sur rocher des Baux, et des reliefs des grands monuments de Basse Provence; mausolée de Saint-Remy ot are d'Orange.

Le panneau sculpté. dont il ne manque que le rouronnement, est roupé i gauche par lo mur de la sacristie et incomplet de la partic droite, qui si? décroche en gradins: la première assise. seule complète à droile. terminée par une plate-bande verticale en forme de pilastre dencadrement. comprend trois blocs (long. de droite ì gauche: 1 m. 5', $1 \mathrm{~m} .47$ et $0 \mathrm{~m}$. 7t. ce dernier masquí sur $0 \mathrm{~m}$. 4i environ); la deuximme. ragalement trois, un petit bloc long de () $\mathrm{m}$. 70 entre deux grands $(1 \mathrm{~m} .27$ ef $1 \mathrm{~m} .30)$ qui étaient sans doute encalrés par deux assises de chaînage disposes en boutisse, qui avaient leur grande face sur la paroi latérale do monument; enfin la lroisione étail formée de trois blocs d'une longueur de $1 \mathrm{~m} .40$ environ, celui de gauche en partie masque et relui de droite manquant. re qui donnail à lassise une longueur le't m. 20 correspondant a la longueur du panneau sculpté.

(2) Le dessin de Millin, auquel nous nous referons (v. p. 170, n. 1) donne comme hallleur des figures 5 pieds 3 pouces, soit $1 \mathrm{~m}$. i0, correspondant i la hauteur des trois assises qui est en réalité de $1 \mathrm{~m} .62$ (et non $2 \mathrm{~m}$. 33, comme l'indique Ch. PiCard, $l$. c., p. 444, n. 2). 
La partio gamedo de re panneau est en effele recouverte par le mur (éj). 0 m. 58) dune pelite salristie, arroléc it l'abside de la chapelle qui fut blevér postéricuroment (atr la tranche de ses assises de base ost visible sous le crépi de la paroi interienere du mur de ladite

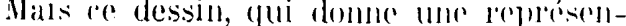
lalion fidele du relief, alver ses mantulues a droite en escalier, ast malloureuscment inexact pour ce mome roble: la première assise du panneall sculphi est raccourcie ef ne romprend pas la platebande verticale, seulptese stlr fo mimn

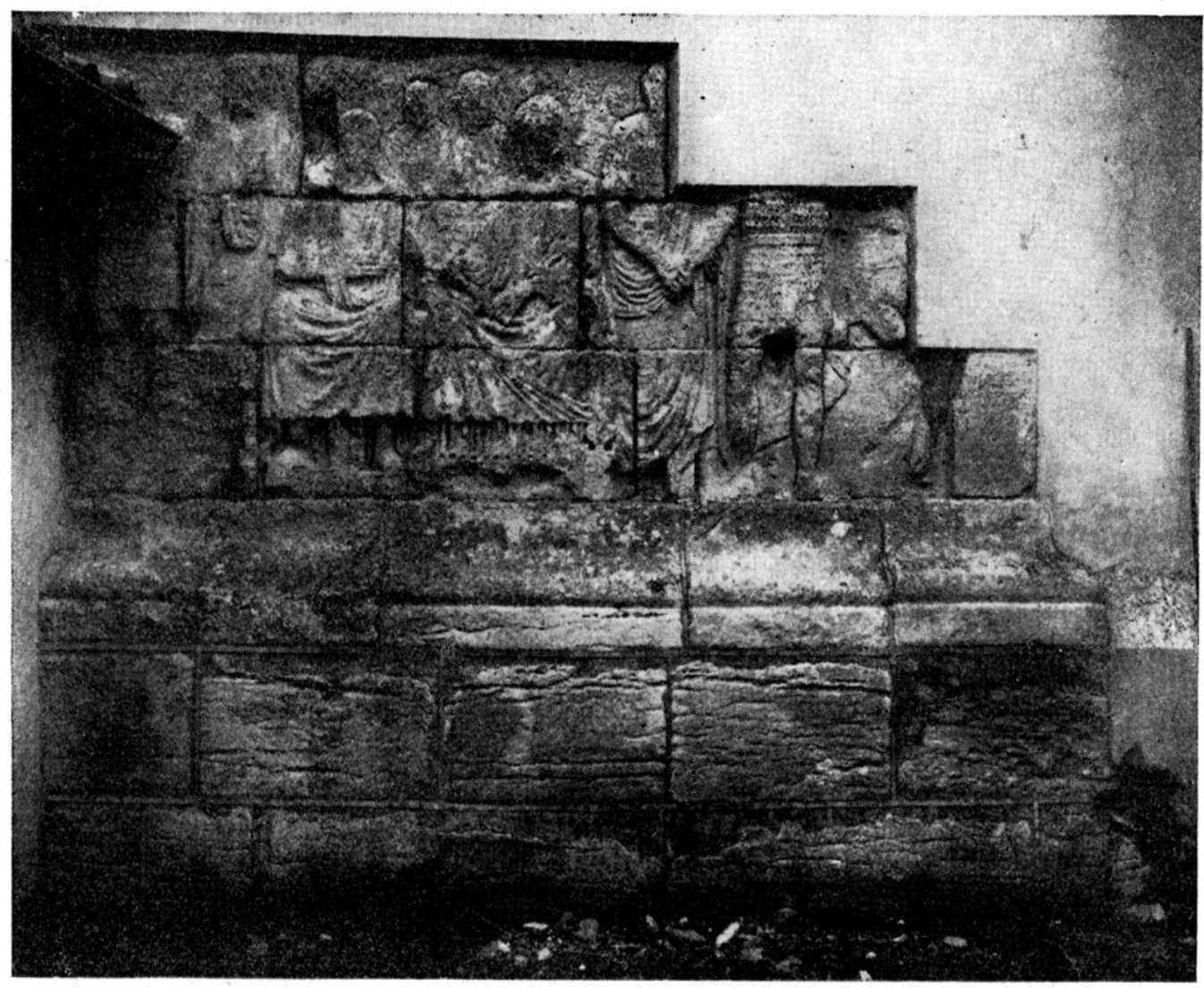

Fif. 1. - Le bas-relief de Sainl-Julien.

(cilinlu: lomulard)

sarpistion. Sil pestitulion mol posible grâte à 111 dessin de Millin, mis vers 1805. aui ronstitue le plus ancien do-

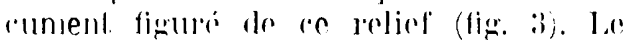

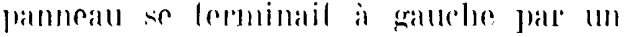

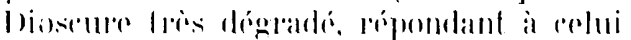

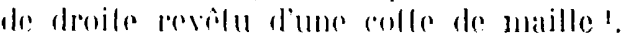

(1) Dessin it lit Bibl. Nal. Ciab. de's listam-

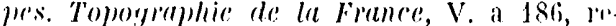
produit par Ch. 1'ICAkn, l. c., p. 441, fig. 2; at grav. dans l'dllas, de Mildus, Voyage dans. les déparlemens du vidi de la France, 1807, pl. LAXI, 6 (nolre fig. 3).

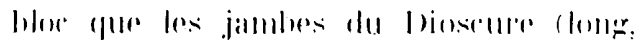

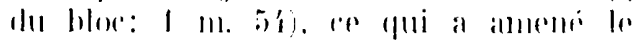

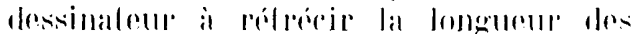

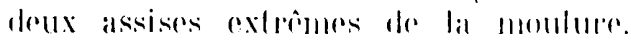
Nul doulde erependand gure lo dessidn de

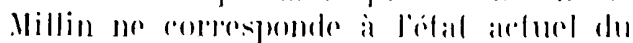
relief: la dimmsion guil rell domme. 1 m.

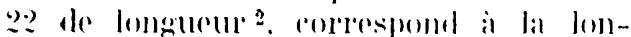
wherur arfuellement visible du relief solt

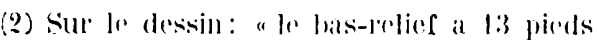
we long", soit a m. 2.216 qui correspond is la longueur 'n coudées el poues ('t m. 21?) indiquée par Ch. Picard, l. c., p. 4.18, no 4. 


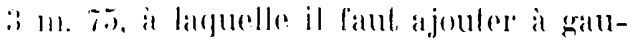
rhe une longueur de $0 \mathrm{~m}$. á envilon

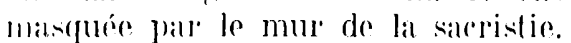

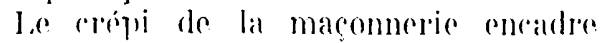

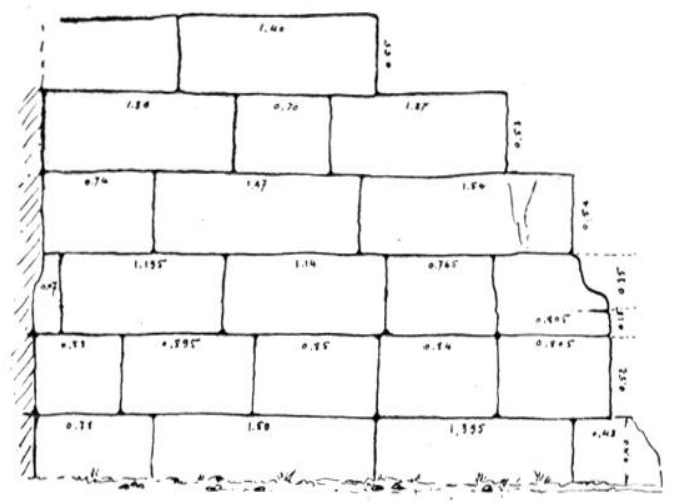

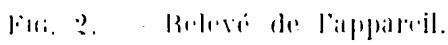

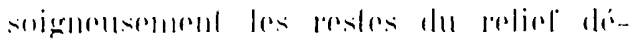

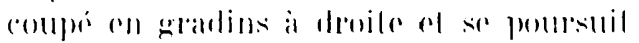

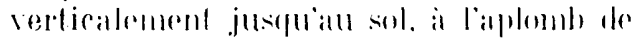
langle moulume du sombatsorment: and a aimsi l'illusion fue la lare no du menulment molato prexistant a la rhapelle,

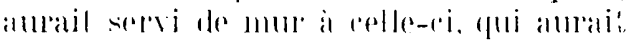
pris alpmi sur les assistes andipules.

Cependant une lablition locale, doul

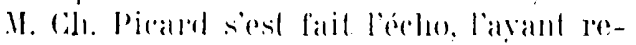

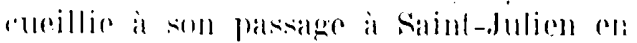

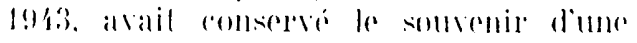

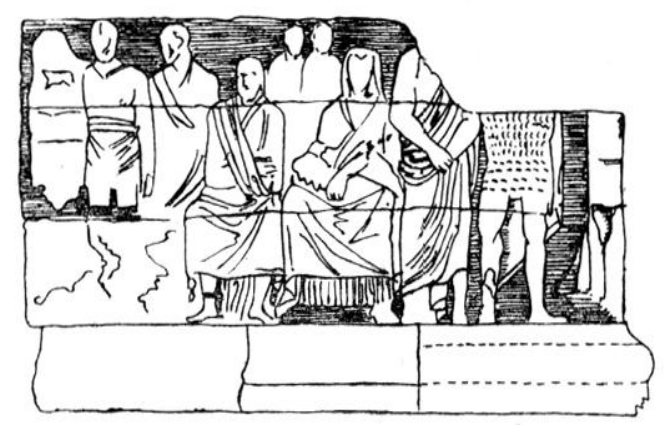

lini. 3. - Jessin do Millin (1805).

mise an place récente du relier, qui anrait rá lamsporté lor's de la construrlion de la chapelle. De fait, on remarque l'épaisseur des joints des assises du relief, lices intéricurement par du mortier

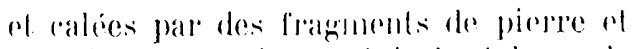
dre luiles noyes dans celui-cie of le modr loul different de liaison des assises du soubassement i joints en relief apparents, non moins que la superposition en "coup de sabre" de rertains joints vertiraux, ce qui est tout a fait anormal dans ume ronstruetion antigue de grand aplia. ril.

Il était interessant de laire me rerifiralion sur le monumont lui-môme. I"ne louille au pied de relui-ci a en effel jortmis de constater que la première assiso du soubassement, all lien te reposer stll tul emmandhement oll une base dmoulurée repondant is la demeine smpépieure.

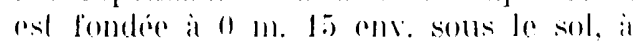
mime le rocher gui nal pas che aplani a cel ofiel. lin olllere, und dos blocs a grosisiomement dié rille jar un pelit cube de pierere; of il ext applitrl, roimer entre le lorher et le morlendu soulatsiement, un limingent de ralolle raitnionno, ne pouvant provenir que da rharnier qui merouvrail au

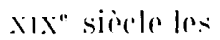

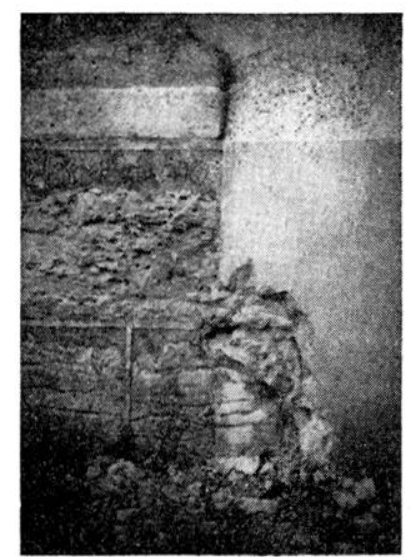

Fuli, i. - Suilu du sollbassement sous te crépi. illorils de la rhapolle.

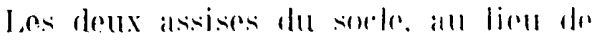

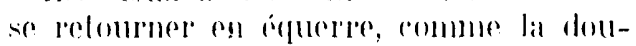
rine gu’il supporle, se prolongent a droil." sous le rerépi du mur jusqu'a la saillie. d'une ehapelle laterale de faroun à former fo mur minse du chorur (fig. 1). 11 blait inulile en re cas de faipe des sondages, a l'interieur de la rhapelle. dansi le mur of sous le dallage du chorur, it lefot de refrourer le prolongement die la paroi latérale de l'ádifice. Ies deux assises du soubassement font partie intaErante du mur de la chapelle qui a elí hati aver des matériaux antiques on remploi; il est indépendant du relief qui a été remonté en cet emplacement, peut- 


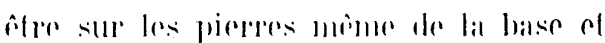
encadre par le répi de revitement afu d'être "présenté" dans ses partios sulpfirs. $\mathrm{xi}$ le relief ni son sorle ne sont dome

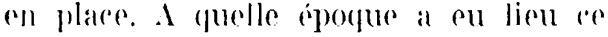
remontage el d'où proviomnent less malíriaux"

les somdages faits sur lo platean all mord a a louest de la rhapelle noml mis all jour alueun vestige antigue. le roce affeurant presefue partoul. lo dessin de Millin se borme a rejpésenter le relied reposint sur sa moulume do hase: il ommel de figurer le soubatsoment. dount il ne donne pas la dimension en hanleur. mais. selon losigge roumant dans les relevés d'arroilecture, il imligure fo profil d'une seronde moulure de base. simplement esquissée au pointilló el non onbrée ${ }^{1}$, opposie a la premirie of qui est absento arturllement. preure sividente que le dessin de Millin a éle fait avant le transfert du relied a sa place actuelle. Mais l'exarfilude du dessin (a part l'erreur de proportion signalée ridessus) pormet de

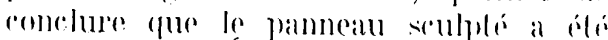

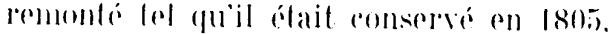

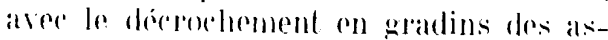

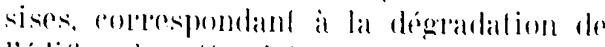
liedifiere a refle dille.

Lal descriplion do Millin esl malhen-

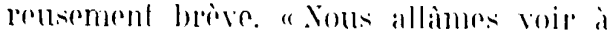

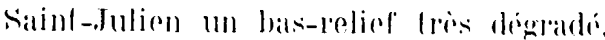
qui déerere du roble nord la mumaille d'une four anligue of presque ruimese "2. Wais. pere aluparavant, l'abbe loulure. ruré de Miramas, a dui l'on doit de prócedenses indicalions sur les antiquiles romaines do la rowion de Martigues of de l'útantr do Berre, etait plus explicite et confir-. mait que le relief faisalt partie dune four of mon d'ume chapelle". "Il serait inferessanl, écril-il, l'avoir la description de la loum on malusolée du Val-Sainl-Julim". Ef " mous avons an Val-silint-

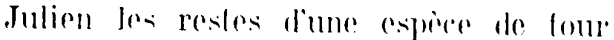
antiguere dont la muraille du nord ast "rmes diun bas-peliof représentanl di-

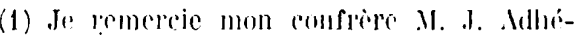
mar. de la veriftention qu'il a bien vonlu faire ill calinet des kstampes.

(2) I"oy!a!/", IN, 1811, p. :3r.

(3) Joum. des sic. et Arls de Harseill' "l rotites affiches du département des Bouchesdu-Rhome. III, nos 633, 10 juillel 1805 ol bs, 5 août 1805. vel: personnagess que linjure du lom

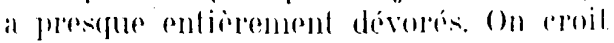

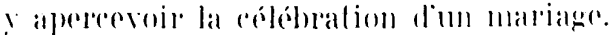
Queleues personnes pensent gur rost un tombealu antiguen.

Nulle mention dans labbe coulume ni dauns Millin do la rhapello Saint-Julien. qui existail rependant a relle datre en ruine, sur la colline dominaml te vallon.

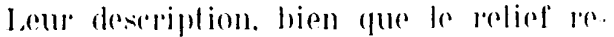
cardal déja le nord dans sa position pumitive. ne concerme done pas lo meme solifice. le monument avait laspered doure four ou d'une "pile". rest-it-dire diun matusolere dont salls doute rail soule

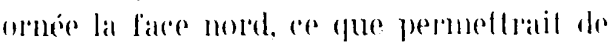
verifier le degagement de la lamehe latlérale de deoite de la premieme assisese.

lin 18:4. par contre. la Stalistique du

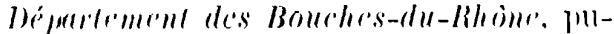
hicé par lo Comle de villeneure. signals yue le bas-lelief fail partie inlégrantr llune rhapelle: "Ia rhapelle Sainl-Juliene dil-il. est ronstruite de malerianx

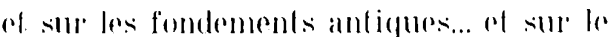
mure septentrional gui regarde lo rime-

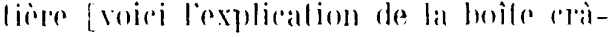

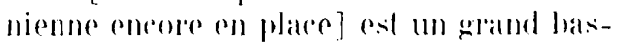
rolief dont les ligures sont de proportions malurello"s. Ia chapelle existail all Woyen àge ainsi quen témoigne mor ryi-

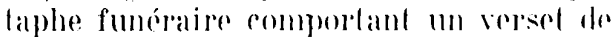

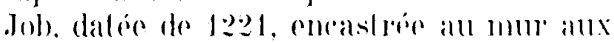
almes de la maisonn didgul, sumajouleres.

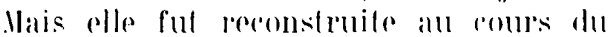
rixe sione par la famille DemandolxDedons. qui possédait lo chateau d'Agul. dans le Val-Saint-Julien. Ine premieres reconstruction doit et re attriburear alus anciens mombres de relle famille. inhulmes dans la rhapelle fumciaiere do la fare mord proche du relief. Jean Gaspard, marquis de Demandolx La Palud (t 1830) of dexandre Eugene Jedons do Pieprefen (t 1858). Ia tradilion lewale. puit a cont-

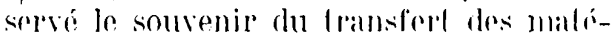
rianx du Val-Saint-Julien, conererne vaisemblablement. un agrandissement de lat chapelle (oonstruetion de lermitage) dont.

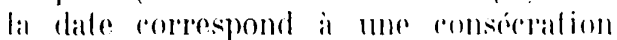
drume staluelle de la Violege le 8 déerenlore 1854. La restaumaton du milien du . reonstruetion romportant to transfert

II, p. 496. 
du bas-relief à une date qui doit itro placée entre 1805 ef 182't. less malerianx avaient été pris aux ruines romaines du Val-Saint-Julien, à l'est du château d'Agut. domaine du marquis Judon de Pierrefeu où la Slatistique de 182.1 signale précisément "les ruines dune villa considérable dans lesquelles on a délerrí plusieurs fragments do statues en marbre et des medailles qui se trouvent dan: le cabinel de 11 . le comle de Panisse "1.

l'exploration du site à 200 mìtres environ au sud de la chapelle en bordure of a l'ouest de la route de Martigues à Sausset section (i de saint-Julien. pareelle 568 a permis de retrouver l'emplatement de cette rilla. Les vestiges forment un monticule inculte. entoure de rhampsi autrefois maréageux, doi ímergent quelques restes de blocige. salls aurume laace de pierre de parement. Dans les ruines, parmi de nombreux fragments de revêtement de marbre, de la poterie de la Graufesengue, une luile aver margue L. Hor(ennii) o $[p t]$ ati de fabrique italique ${ }^{2}$ et un hlor quadrangulaire on pierre de la couronne, avant pu apparte. nir au monument funéraire.

Ces ruines romportaient un idifice fumeraire. In el peul-être deux sarrophages en proviennent: l'un pll pierre ser'vant de pile a un puits, a 250 moldes a lest de ce sile (long. I m. 90); larg. 0 II 65; haut. (1 m. 35), sans alucume deroration. mais portant sur la tranche supérieure des parois une rainure jour la fermeture du rouverele: le second serait dans une collection privere de Sillsisel.

peut-rtre les fouilles faites a lemplatrement de ces substructions permettraitalles de relrouver, sinon les assises du monument en pierre de la Couromne. rui ont dù ôtre employées comme matériaux. du moins.le dessin des fondations, qui en donnerait le plan.

\section{Fernand Bexoltr.}

(1) II, p. 966 ; F.O.R., 154.

(2) Les tuiles de cette fabrique sont en effet particulièrement nombreuses dans la région de Vintimille, Nice, Cimiez el préjus: CIL, V, 8110, 445; XII, 5679, 44. Cf. II. C.uerc, Massalia, II, p. 310, n. 2. Ces fragments ont été déposés all Musée du VieuxMartigues.

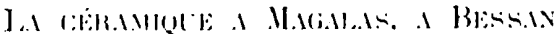

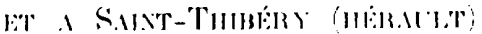

Duranl la pépiode de 1928 a 1938, noms avons en la bonne fortune de decouviris Irois stations préromaines gui nous ont donné quelpues fessons ibépigues. les résultals les plus interessants nous ont áté fournis par lonppielum de llonlfo joris de Magalas silue a lo kilomedres all nord de Beziers of a ?h kilomides a l'ourstnorl-ouest d'Ensirmes.

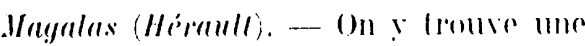
slatigraphie remarefuable qui presente parfois a melres do andehes areheologiques en plare. Elle a permis de dater ajeproximativement les lessons de poleride en remonlant depuis le 1er sionde de notor

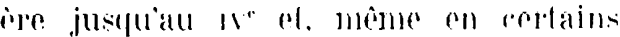

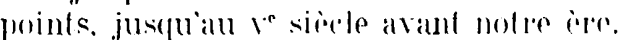

Yous rencontrons sucessivement: la polerie do la cimulesenque. la réramifue de lanlatisie dimportation demaine (la lechnique d'Arezzo est également re-

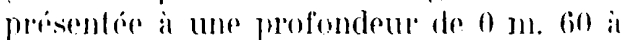
I motres: la polerie campanieme ensuite. mólangée a la polerie indigène. Tous ces débris de vases ou de coupes sont areommagness de monnaies de plus ch plus anciennes a mesture quion approfondit la fontille. ${ }^{\circ}$ A la polerie de la (imalesencue do surfare romespondent

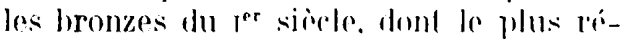
rent est le Domilien. Ces monnaies gisent

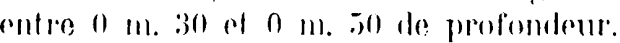
". Aver la réramique arrétine apparais-

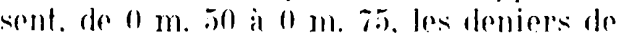
la Republique ef les piòes de Diane nu d'Apollon frappess a Marseille. $3^{\circ}$ Plus has au niveau de 0 m. 35 a 1 metre. se reneontrent on plus grand nomber les hromzes de Mnessilin aver la réramique

3) Cf. mense auleur: Wugalas ot son oppithem de Vontfo, dans congres préhistorique de France, 1936 39 p.): La slation grecque do la Monedière, pròs Bessall llléraull), dans Cahiers d'histoire et darchéologie, Nìmes, 1936 (23 p.): L'oppidum et les atelier's de lontfo, rontinuation des fouilles, dans Confrès de l'association francaise pour l'aramrement des sciences, Marseille, 1936 (3 p.); L'oppidum el les ateliers de Montfo près Maaqalas, dans Bull. archérl. du Comité, 193619.3\%, pp. 431-43.4. 